\title{
A paradoxical risk aversion effect on the consumers' demand for quality
}

\author{
David Bardey* \\ Gremaq, Université Toulouse $I^{* *}$
}

\section{Introduction}

In most vertical differentiation models, the quality of the products is a known variable and enters the utility function as a deterministic variable. Nevertheless, some recent contributions, Krishna and Winston (2000) and Grilo and Wauthy (2000), interpret the quality issue as a probability that the product "delivers to the consumer the satisfaction he expected to enjoy". These articles study the impact of quality uncertainty on market structure. In this paper, we restrict their quality concept to the "survival probability" of the product in order to focus on the quality demand function. In fact, for a wide class of products such as durable goods, the consumers often decide to buy more expensive products in order to be "more sure" that they will not break down (or last longer). The survival probability of a product being one important dimension of its quality, demand for quality crucially depends on the risk aversion that characterizes the consumers' preferences.

In this paper, we fill this gap in the literature. We use the framework of vertical differentiation models to analyze the consequences of buyers' risk aversion. ${ }^{1}$ In the discrete version of this class of models, two kinds of consumers are distinguished : the high valuation and the low valuation ones.

* I thank Anne Perrot, Philippe Choné, Maia David, Maiseu Dumans, Jorge Ferrando, Romain Lesur, Laurent Linnemer and Michael Visser for their comments and remarks. l'd like to thank the anonymous referee of the review for his helpful comments.

** UMR CNRS 5604, Université des Sciences Sociales, Manufacture des Tabacs, Bat F - 2ème étage, Bureau 207, 21 Allée de Brienne, 31000 Toulouse, France. E-mail : david.bardey@univ-klse1.ir

1 Even though Grilo and Wauthy (2000) have used a continuous distribution for consumers' risk aversion, they use certainty equivalent, which does not permit to capture a risk aversion effect on demand for quality. 
Naturally, the high valuation consumers are characterized by a higher willingness to pay for the good. The seller cannot observe this characteristic, which constitues a private information of the buyers, and he/she must therefore introduce a second-order price discrimination in order to extract (more) consumers' surplus.

Intuitively, we could imagine that if high valuation consumers are more risk averse than the low valuation ones, they would like to buy the higher quality product in order to reduce the probability that the good breaks down. ${ }^{2}$ So, in a vertical differentiation model, this second source of heterogeneity between consumers should a priori not change the incentive problem and the high valuation incentive constraints may be relaxed. Contrary to this intuition, we prove in the section 2 that if high valuation consumers are also more risk averse, then the traditional second-order discrimination mechanism may paradoxically be disturbed by a risk-aversion effect. We conclude in the third section.

\section{Uncertainty around demand for quality and risk aversion}

In the first subsection, we use the framework of Mussa and Rosen (1978) and we explain that, in their model, quality can also be interpreted as a probability. In the second subsection, we study the incentive constraints when high valuation consumers are more risk averse. We make the link between consumer's demand for quality and self-protection theory propounded by Dionne and Eeckhoudt (1985) in the third subsection.

\subsection{Quality and probability of survival in a vertical differentiation model}

Consider the simplest discrete version of the Mussa-Rosen vertical differentiation model with two types of consumers, characterized by different levels of their taste parameter : $\vartheta_{i}$ with $i=\{H, L\}$ and $\vartheta_{H}>\vartheta_{L}$. The proportions of high and low valuation consumers are respectively $\lambda$ and $1-\lambda$. In their model, consumers are assumed to be risk neutral, and the consumer $i$ utility function is :

$$
U_{i}=\vartheta_{i} q-P
$$

where $q$ and $P$ are respectively the quality level of the product and the price paid to the seller. We remark that this utility function can be interpreted as

2 Unfortunately, analysis which deal with quality do not consider that quality is an increasing function of the survival probability of the product. If the reader wants to be convinced by this intuition, after explaining that quality increases the survival probability, he can ask his colleagues the following question : "For a same taste, if you are more risk averse than me, will you buy higher quality ?" Most of the time, the answer is positive. 
an expected utility if $q$ is viewed as a probability that the product generates a satisfaction $\vartheta_{i}$ and while with a probability $1-q$ it generates 0 . Indeed,

$$
U_{i}=q\left(\vartheta_{i}-P\right)+(1-q)(-P)
$$

which is equivalent to (1). Since the quality becomes the survival probability of the product, in the good state of the world, the product generates a satisfaction $\vartheta_{i}$ less the price paid, whereas in the bad state the product breaks down and the consumer's utility is simply the opposite of the price paid.

The seller's problem is to maximize his profit. For simplicity, we assume that his cost function ${ }^{3}$ is $c(q)=\frac{q^{2}}{2}$. Since the seller cannot observe the consumers' type, he/she must offer two contracts for which the consumers self-select according to their taste parameter.

So the seller program is :

$$
\left\{\begin{array}{l}
\max _{\left(q_{H}, q_{L}, P_{H}, P_{L}\right)} \lambda\left(P_{H}-\frac{q_{H}^{2}}{2}\right)+(1-\lambda)\left(P_{L}-\frac{q_{L}^{2}}{2}\right) \\
s . t \\
\vartheta_{H} q_{H}-P_{H} \geqslant \vartheta_{H} q_{L}-P_{L} \quad(I C H) \\
\vartheta_{L} q_{L}-P_{L} \geqslant \vartheta_{L} q_{H}-P_{H} \quad(I C L) \\
\vartheta_{H} q_{H}-P_{H} \geqslant 0 \quad(P C H) \\
\vartheta_{L} q_{L}-P_{L} \geqslant 0 \quad(P C L)
\end{array}\right.
$$

Where ICH and ICL are the incentive constraints associated to types $\mathrm{H}$ and $\mathrm{L}$, and PCH and PCL the usual participation constraints. The secondorder price discrimination schemes are well-known : the high valuation consumers incentive constraints ( $\mathrm{ICH}$ ) and the low valuation consumers participation constraints (PCL) are binding. Then, the second-best contracts $c^{H}=\left(q_{H}, P_{H}\right)$ and $c^{L}=\left(q_{L}, P_{L}\right)$ are :

$$
\begin{aligned}
q_{H} & =\vartheta_{H} \\
P_{H} & =\frac{\vartheta_{H}^{2}+\vartheta_{L}^{2}-(1+\lambda) \vartheta_{H} \vartheta_{L}}{(1-\lambda)}
\end{aligned}
$$

for high valuations consumers, and

$$
\begin{aligned}
q_{L} & =\frac{\vartheta_{L}-\lambda \vartheta_{H}}{1-\lambda} \\
P_{L} & =\frac{\vartheta_{L}\left(\vartheta_{L}-\lambda \vartheta_{H}\right)}{1-\lambda}
\end{aligned}
$$

3 We use this assumption in order to be as close as possible to the Mussa-Rosen framework. However, Deneckere and Mc Afee (1996) give several examples for durable goods where low quality is more costly. It could be also interesting to study the risk aversion effect with their assumption. 
for the low valuations ones.

High valuation consumers receive the first-best quality level whereas the quality is reduced for the low valuation consumers in order to guarantee the high valuation incentive constraints.

\subsection{Risk aversion heterogeneity between consumers and second-order price discrimination}

Now, assume that the high valuation consumers are more risk averse than the low valuation ones. This assumption seems to be realistic ${ }^{4}$ and only implies that the group of consumers who have a higher willingness to pay for the product are also more afraid that the product breaks down.

We consider that risk aversion of the high valuation consumers can be represented by a concave transformation $(k)$ of $U$ (Pratt (1964), KihlstromMirman (1974)). Here, we restrict our attention to a quadratic utility function: $V(W)=E\left[W-\gamma W^{2}\right]$ where the degree of risk aversion is captured by $\gamma .^{5}$ Then, the expected utility of high valuation agents who pay a price $P$ to obtain a quality $q$ is :

$$
\begin{aligned}
V_{H} & =q\left(\left(\vartheta_{H}-P\right)-\gamma\left(\vartheta_{H}-P\right)^{2}\right)+(1-q)\left(-P-\gamma(-P)^{2}\right) \\
& =U_{H}-\gamma\left(q \vartheta_{H}^{2}+P^{2}-2 \vartheta_{H} q P\right)
\end{aligned}
$$

Our objective is to check whether the incentive constraint (ICH) is still respected with the previous menu $\left(c^{H}, c^{L}\right)$ since high valuation consumers are now risk averse. It is indeed the case if :

$$
V_{H}\left(c^{H}, \vartheta_{H}\right) \geqslant V_{H}\left(c^{L}, \vartheta_{H}\right)
$$

The last condition is verified when :

$$
\begin{array}{r}
U_{H}\left(C^{H}, \vartheta_{H}\right)-\gamma\left(q_{H} \vartheta_{H}^{2}+P_{H}^{2}-2 \vartheta_{H} q_{H} P_{H}\right) \geqslant \\
U_{H}\left(C^{L}, \vartheta_{H}\right)-\gamma\left(q_{L} \vartheta_{H}^{2}+P_{L}^{2}-2 \vartheta_{H} q_{L} P_{L}\right)
\end{array}
$$

(ICH) is binding so we have $U_{H}\left(C^{H}, \vartheta_{H}\right)=U_{H}\left(C^{L}, \vartheta_{H}\right)$ and the last condition becomes :

$$
\left(q_{H} \vartheta_{H}^{2}+P_{H}^{2}-2 \vartheta_{H} q_{H} P_{H}\right) \leqslant\left(q_{L} \vartheta_{H}^{2}+P_{L}^{2}-2 \vartheta_{H} q_{L} P_{L}\right)
$$

Using (6), (7), (8) and (9), (12) becomes :

$$
\vartheta_{L}^{2}\left(1-3 \lambda-2 \lambda^{2}+2 \lambda \frac{\vartheta_{L}}{\vartheta_{H}}\right)+\vartheta_{L}\left(2 \lambda^{2}-1+\lambda\right) \leqslant(1-\lambda) \vartheta_{H}\left(\vartheta_{H}-1\right)
$$

\footnotetext{
4 Especially for durable goods. If someone watches TV a lot (so he has a high willingness to pay for a TV), he is probably more risk averse about the break-down of the TV than consumers who don't often watch TV.

5 Where $(k)$ is such that $k^{\prime}>0, k^{\prime \prime}<0$ and $k^{\prime}=\frac{V^{\prime}}{L^{\prime \prime}}=\frac{(1-2 \gamma W)}{(1-2.0 W)}=1-2 \gamma W$.
} 
This leads to the following proposition.

Proposition 1 In a second-order price discrimination model with two types of consumers, if high valuation agents are more risk averse, whatever the degree of the risk aversion, and if the seller does not take into account the risk aversion heterogeneity among consumers, the high valuation agents may prefer the quality destined to the low ones.

It is very easy to verify that the last condition can be not satisfied for very realistic values of the parameters. If we take the proportions of the two consumers types equal to $1 / 2, \vartheta_{H}=0.6$ and $\vartheta_{L}=0.4$, we have $V_{H}\left(c^{H}, \vartheta_{H}\right)<V_{H}\left(c^{L}, \vartheta_{H}\right)$. The more important point of the proposition, is that this incentive constraint is violated whatever the degree of risk aversion. If high valuation consumers' degree of risk aversion are infinitesimal, the second-order price discrimination can be disturbed.

The lower is the value of $\vartheta_{L}$, the lower the value of $\vartheta_{H}$ has to be to violate the incentive constraints of high valuation consumers when they are risk averse. When the proportion of high valuation consumers increases, the step value of $\vartheta_{H}$ for which $V_{H}\left(c^{H}, \vartheta_{H}\right)<V_{H}\left(c^{L}, \vartheta_{H}\right)$ increases too. It is due to the fact that for optimal contracts under risk neutrality, all things equal, the difference $P_{H}-P_{L}$ increases with the proportion $\lambda$ of $\mathrm{H}$-type consumers. According to the fact that for the H-type, the quality is equal to the taste parameter (i.e $q_{H}=\vartheta_{H}$ ) and that the price $P_{H}$ is increasing with $\vartheta_{H}$, when the proportion $\lambda$ increases, the parameter $\vartheta_{H}$ (so the quality sold to the H-type consumers) must be not too high to insure the incentive constraint, so to avoid that the risk aversion effect linked to the price paid becomes too strong.

Of course, from a methodological point of view, it can appear usual that optimal allocations derived in a monodimensional setting are not robust in a multidimensional framework. However, we guess that the risk aversion effect generated by the uncertainty dimension of the quality was until then implicitly neglected because of the wrong intuition that positive correlation between risk aversion and valuation of the quality favors the relaxing incentive constraints instead of violating them.

Remark 1 In a model which takes into account the heterogeneity of risk aversion, the paradoxical effect of risk aversion favors bunching allocation.

According to the paradoxical effect of risk aversion, high valuation consumers may prefer lower quality due to the uncertainty dimension of the quality.

\subsection{Demand for quality and self-protection theory}

The consumers' demand for quality and the self-protection theory follow the same mechanism. Eeckhoudt and Dionne (1985) showed that self-protection 
activities may paradoxically decrease when agents are more risk averse. ${ }^{6}$ Briys-Schlesinger (1990) offered a very intuitive interpretation of this paradoxical risk aversion effect for self-protection actions that we can apply to the quality choice problem. If we reason with the final wealth distribution, a higher quality does not imply a less risky distribution. ${ }^{7}$ To understand this phenomenon, imagine you decide to buy an expensive TV in order to reduce the break down probability of your new television set. If unfortunately your television set breaks down anyway, you are in the worst situation : you spent a lot of money and you have no television. Surprisingly, this mechanism, now well known in the self-protection theory, is not amazingly considered in the quality literature.

\section{Conclusion}

In this note, we focus on a very usual trade-off made by consumers each time they buy a durable good: "If I pay more, the break down probability is reduced". We study the consequences of this trade-off on the secondorder price discrimination policy. An intuitive idea is that more risk-averse consumers will prefer to pay a more expensive price. We explain in this note that demand for quality corresponds to a similar choice for self-protection activities and we simply apply the self-protection theory to analyze the demand for quality specificity. If a consumer pays a higher price but the good breaks down anyway, he is in the worst situation. This mechanism is not monotone with risk aversion which implies very strong difficulties to develop a multidimensional model where consumers are both represented by their quality valuation and their degree of risk aversion. This work is a first step and only allows to conclude that risk aversion favors bunching allocation.

6 See also Eeckhoudt and al. (1997) for an analysis of risk aversion effect on willingness to pay and on premium risk.

7 To be more specific, Briys and Schlesinger (1990) proved that a higher self-protection decision does not imply a less risky distribution in the second order dominance stochastic. 


\section{Bibliographie}

Briys E. and H. Schlesinger (1990), "Risk Aversion and the Propensities for Self-Insurance and Self-Protection", Southern Economic Journal, 57, 2, pp.458-467.

Deneckere R. and P. Mc Afee (1996), "Damages Goods", Journal of Economics and Management Strategy, 5, pp.149-174.

Eeckhoudt L. and G. Dionne (1985), "Self-Insurance, Self-Protection and Increased Risk Aversion", Economics Letters, 17, pp.39-42.

Eeckhoudt L., Ph. Godfroid and C. Gollier (1997), "Willingness to pay, the Risk Premium and Risk Aversion", Economics Letters, 55, pp.355-360.

Grilo I. and X. Wauthy (2000), "Price Competition when Product Quality is Uncertain", Recherches Economiques de Louvain, 66(4), pp.415-437.

Kihlstrom R. and L. Mirman (1974), "Risk Aversion with many commodities", Journal of Economic Theory, 8, pp.361-388.

Krishna K. and T. Winston (2000), "If at first you don't succed... Profits, Prices and Market Structure in a Model of Quality with Unknowable Consumer Heterogeneity", NBER Working Paper Series.

Laffont J-J. and D. Martimort (2002), Theory of Incentives : The PrincipalAgent Model, in progress.

Mussa M. and S. Rosen (1978), "Monopoly and Product Quality", Journal of Economic Theory, 18, pp.201-317.

Pratt J. (1964), "Risk Aversion in the Small and in the Large", Econometrica, pp.122-136. 
